\title{
SHARP ISOPERIMETRIC INEQUALITIES FOR STATIONARY VARIFOLDS AND AREA MINIMIZING FLAT CHAINS $\bmod k$
}

\author{
JAIGyOUNG CHOE*
}

It is well known $[4,5,8]$ that a smooth minimal surface $\Sigma$ spanning a rectifiable Jordan curve $C$ satisfies the isoperimetric inequality

$$
4 \pi \operatorname{Area}(\Sigma) \leqq \text { Length }(C)^{2},
$$

where equality holds if and only if $C$ is a circle and $\Sigma$ is a disk. Some smooth minimal surfaces in $\boldsymbol{R}^{3}$ can be physically realized as soap films. However, the soap films formed by dipping some wire frames in soap solution contain interior singular curves. Here arises the main question of this paper: Does (1) still hold for this soap-film-like surface with singularities? In 1986 one of Almgren's results [2] answered this question affirmatively for area minimizing flat chains $\bmod k$. In this paper we extend his two-dimensional result and show that (1) holds also for two-dimensional stationary varifolds with connected boundary of multiplicity $\geqq 1$ (Theorem 2 ). Moreover, if the bounding curve $C$ consists of $k$ curves having the same end points, we obtain a new type of sharp isoperimetric inequality for area minimizing flat chains $\bmod k$ spanned by $C$. Here, unlike (1), equality holds only for the union of $k$ flat half disks with a common diameter (Theorem 3).

\section{Ares and sectors}

In this section we derive sharp isoperimetric inequalities for domains in the plane where only a specific part of the boundary counts toward the length of the boundary.

LEMMA 1. Let $l_{1}$ and $l_{2}$ be the rays emanating from a point $O$ with an angle of $\theta \leqq \pi$. Let $C$ be a curve from a point of $l_{1}$ to a point of $l_{2}$.

(a) Suppose that $C$ lies in the smaller sector of the two formed by the rays ( $C$ may lie in either sector if $\theta=\pi$ ). Define $D$ as the domain bounded by $l_{1}, l_{2}$, and $C$. Then

* This work was partially supported by GARC and KOSEF (921-0100-001-2).

Received January 30, 1995, revised June 19, 1995. 
$2 \theta$ Area $(D) \leqq$ Length $(C)^{2}$,

and equality holds if and only if $C$ is a circular arc perpendicular to the rays.

(b) If $C$ lies in the larger sector, then

$$
2 \pi \text { Area }(D) \leqq \text { Length }(C)^{2},
$$

where equality holds if and only if $C$ is a semicircle perpendicular to one of the two rays.

Proof. (a) For the existence of an optimal curve of fixed length enclosing the largest area, we refer to [10, pp. 441-444]: the set $S$ of convex curves of fixed length lying in the smaller sector, being equipped with the topology corresponding to the Hausdorff metric, is compact; the two functions defined on $S$, Length and Area, are continuous. Let $C$ be an optimal curve which is convex and lies in the smaller sector, and let $p_{i} \in l_{\imath}, i=1,2$, be the end points of $C$. We claim that the triangle $O p_{1} p_{2}$ is an isosceles triangle. If it is not, choose $p_{i}^{\prime} \in l_{2}$ such that $O p_{1}^{\prime} p_{2}^{\prime}$ is an isosceles triangle with Length $\left(\overline{p_{1}^{\prime} p_{2}^{\prime}}\right)=$ Length $\left(\overline{p_{1} p_{2}}\right)$. Let $C^{\prime}$ be the curve from $p_{1}^{\prime}$ to $p_{2}^{\prime}$ which is congruent to $C$. Then the domain $D^{\prime}$ enclosed by $C^{\prime}$ and $l_{1}, l_{2}$ has larger area than $D$. If $C^{\prime}$ lies in the smaller sector, the existence of $C^{\prime}$ contradicts the optimality of $C$, and so our claim follows. Suppose a part $C^{\prime \prime}$ of $C^{\prime}$ leaves the smaller sector. Let $p^{\prime} \in l_{1} \cup l_{2}$ be the end point of $C^{\prime \prime}$ which is not an end point of $C^{\prime}$. Rotate $C^{\prime \prime}$ around $p^{\prime}$ by $180^{\circ}$ and obtain a curve $\bar{C}$ in the smaller sector. Then $\left(C^{\prime} \sim C^{\prime \prime}\right)$ $\cup \bar{C}$ is a nonconvex curve of the same length as $C$ in the smaller sector which encloses the same area as $C$. Hence one could find a convex curve of smaller length enclosing larger area than $C$, which is again a contradiction. Therefore $O p_{1} p_{2}$ must be an isosceles triangle.

Next we claim that $C$ is a circular arc. $C$ lies on one side of the straight line $l$ containing $p_{1}$ and $p_{2}$. Find a circle $\gamma$ through $p_{1}$ and $p_{2}$ such that an arc $\alpha$ of $\gamma$ from $p_{1}$ to $p_{2}$ has the same length as $C$ and lies on the same side of $l$ as $C$. Then the closed curve $(\gamma \sim \alpha) \cup C$ gives a solution to the classical isoperimetric problem. The uniqueness of the solution implies $C=\alpha$.

So we have infinitely many candidates for the optimal curve: circular arcs of the same length that are perpendicular to the ray $l_{3}$ which bisects the smaller sector formed by $l_{1}$ and $l_{2}$. We claim that the arc we want should be perpendicular to the rays $l_{1}$ and $l_{2}$ too. For any circular arc $A$ perpendicular to $l_{3}$, $l_{3}$ divides $A$ into two subarcs, $A_{1}$ and $A_{2}$. Move $A_{1}$ and $A_{2}$ separately to the opposite side of $l_{3}$ by the rotations about $O$ by the angles of $\theta / 2$ and $-\theta / 2$. Then we obtain a curve $A^{\prime}$ in the sector which is of the same length as $A$ and encloses the same area as $A$ does. If $A$ is not perpendicular to $l_{1}$ and $l_{2}$, then $A^{\prime}$ is not smooth at $A^{\prime} \cap l_{3}$ and hence $A^{\prime}$ cannot enclose maximal area. Thus the circular arc perpendicular to $l_{1}$ and $l_{2}$ and only this arc maximizes the area of the domain bounded by $l_{1}$ and $l_{2}$. Since this arc satisfies $2 \theta \operatorname{Area}(D)=$ Length $(C)^{2}$, the desired inequality follows. 
(b) Given any curve $C$ joining $l_{1}$ to $l_{2}$ in the larger sector, one can increase the enclosed area of the domain bounded by $l_{1}, l_{2}$ and $C$ by moving (rotating and translating) $C$ into a curve $C^{\prime}$ in such a way that both the end points of $C^{\prime}$ lie on $l_{1}$. Therefore part (a) completes the proof.

\section{Cones with vertex on the boundary}

Some two-dimensional cones in $\boldsymbol{R}^{n}$ satisfy the classical isoperimetric inequality (see [5, Theorem 1]). This follows from the fact that two-dimensional cones, being flat, can be flattened (i.e., developed) to become a planar domain provided its density at the vertex is not smaller than 1 . However, if the vertex lies on the boundary of the cone, the density hypothesis can be dropped [5, Corollary 1].

DEFINITION 1. i) A compound Jordan curve is a one-dimensional rectifiable connected set in $\boldsymbol{R}^{n}$ which is the union of finitely many Jordan curves (=homeomorphic images of a circle).

ii) For $p \in \boldsymbol{R}^{n}, p \times C$ is the cone from $p$ over $C$, the set of all line segments from $p$ to the points of $C$.

Lemma 2. If $C$ is a compound Jordan curve in $\boldsymbol{R}^{n}$ and $p$ a point of $C$, then

$$
4 \pi \text { Area }(p \times C) \leqq \text { Length }(C)^{2} .
$$

Equality holds if and only if $p \times C$ can be developed, by cutting and inserting, one-to-one onto a disk.

Proof. Choose a point $q$ from $C$ such that its distance from $p$ is the maximum among all points of $C$. One can easily find a pair of curves $\gamma^{\prime}, \gamma^{\prime \prime} \subset C$ joining $p$ to $q$ and intersecting each other at finitely many points. Let $\gamma_{1}=$ $\gamma^{\prime} \cup \gamma^{\prime \prime}$. Take $\gamma_{2}, \gamma_{3}, \cdots, \gamma_{k} \subset C$ to be pairwise disjoint curves such that

$$
C=\bigcup_{i=1}^{k} \gamma_{\imath} \text { and Length }(C)=\sum_{i=1}^{k} \operatorname{Length}\left(\gamma_{\imath}\right)
$$

Now let us define a canonical parameter $\theta$ for each $\gamma_{\imath}$. Let $l_{i}(\theta)=\operatorname{dist}\left(p, \gamma_{i}(\theta)\right)$. The desired canonical parameter $\theta$ is assumed to satisfy the condition that

$$
\operatorname{Area}\left(p \times \gamma_{i}([a, b])\right)=\frac{1}{2} \int_{a}^{b} l_{i}^{2}(\theta) d \theta \text { for any } \gamma_{i}(a), \gamma_{i}(b) \in \gamma_{i} \text {. }
$$

From this condition one can easily understand that $\theta$ is the angle parameter viewed from $p$. Assume that each curve $\gamma_{i}(\theta)$ is defined on an interval $\left[0, a_{\imath}\right]$, and assume $\gamma_{1}(0)=\gamma_{1}\left(a_{1}\right)=p$. But $\gamma_{i}(0), i \geqq 2$, may or may not coincide with $\gamma_{i}\left(a_{\imath}\right)$. With each $\gamma_{2}$ can one associate a curve $C_{2}$ on a plane $I I$ which is the polar graph of $l_{i}(\theta)$ : more precisely, $C_{\imath}$ is the set of all points $\left(l_{i}(\theta), \theta\right)$ in polar coordinates on $\Pi$. Then 


$$
\text { Length }\left(\gamma_{\imath}\right)=\operatorname{Length}\left(C_{\imath}\right), \quad \operatorname{Area}\left(p \times \gamma_{\imath}\right)=\operatorname{Area}\left(O \otimes C_{\imath}\right),
$$

where $O$ is the origin of $\Pi$. Geometrically, one can obtain $O \otimes C_{\imath}$ by developing $p \otimes \gamma_{i}$ on $\Pi$, cutting $p \times \gamma_{i}$ along the line segment $\overline{p \gamma_{i}(0)}$ if $\gamma_{i}(0)=\gamma_{i}\left(a_{\imath}\right)$.

Without loss of generality one can assume that $a_{\imath} \leqq \pi$ for all $i \geqq 2$, for otherwise one can divide $\gamma_{2}$ into smaller parametrized curves with the desired property. By Lemma 1(a) there exists a circular arc $A_{\imath}, i \geqq 2$, on the sector formed by $O C_{\imath}$ such that

$$
\operatorname{Area}\left(O \circledast C_{\imath}\right)=\operatorname{Area}\left(O \circledast A_{\imath}\right), \quad \text { Length }\left(C_{\imath}\right) \geqq \operatorname{Length}\left(A_{\imath}\right) \text {. }
$$

Note that since $q$ is a maximum distance away from $p$,

$$
\operatorname{dist}\left(O, A_{\imath}\right) \in\left\{\operatorname{dist}(O, m): m \in C_{i}\right\}=\left\{\operatorname{dist}(p, s): s \in \gamma_{i}\right\} \subset\left\{\operatorname{dist}(p, r): r \in \gamma_{1}\right\} .
$$

Hence there exists $b_{i} \in\left(0, a_{1}\right), i \geqq 2$, such that $l_{1}\left(b_{i}\right)=\operatorname{dist}\left(O, A_{\imath}\right)$. Renumbering $\gamma_{2}$ if necessary, we can assume $b_{2} \leqq b_{3} \leqq \cdots \leqq b_{k}$. Define a new function $l:\left[0, \sum_{\imath=1}^{k} a_{\imath}\right] \rightarrow \boldsymbol{R}$ by

$$
\begin{aligned}
& l(\theta)=l_{1}(\theta), \quad \text { if } 0 \leqq \theta \leqq b_{2} \\
& l(\theta)=l_{1}\left(b_{2}\right), \quad \text { if } b_{2} \leqq \theta \leqq b_{2}+a_{2} \\
& l(\theta)=l_{1}\left(\theta-a_{2}\right), \quad \text { if } b_{2}+a_{2} \leqq \theta \leqq b_{3}+a_{2} \\
& l(\theta)=l_{1}\left(b_{3}\right), \quad \text { if } b_{3}+a_{2} \leqq \theta \leqq b_{3}+a_{2}+a_{3} \\
& l(\theta)=l_{1}\left(\theta-\sum_{\imath=2}^{j-1} a_{\imath}\right), \quad \text { if } b_{j-1}+\sum_{\imath=2}^{j-1} a_{\imath} \leqq \theta \leqq b_{j}+\sum_{\imath=2}^{j-1} a_{\imath} \\
& l(\theta)=l_{1}\left(b_{j}\right), \quad \text { if } b_{j}+\sum_{\imath=2}^{j-1} a_{\imath} \leqq \theta \leqq b_{j}+\sum_{\imath=2}^{3} a_{\imath} \\
& l(\theta)=l_{1}\left(\theta-\sum_{\imath=2}^{k} a_{\imath}\right), \quad \text { if } \max b_{j}+\sum_{\imath=2}^{k} a_{\imath} \leqq \theta \leqq \sum_{\imath=1}^{k} a_{\imath} .
\end{aligned}
$$

Geometrically, the cone $O \notin \bar{C}, \bar{C}$ being the polar graph of $l$ on $\Pi$, can be obtained from $O \otimes C_{1}$ by cutting out the line segments $\overline{O C_{1}\left(b_{i}\right)}$, widening these gaps by the angle of $a_{\imath}$, and inserting $O \circledast A_{\imath}$ in each gap. (See [5, Theorem 1] and $[6$, Lemma 4$]$ for similar arguments.)

In the construction of $l$ above, we tacitly assumed that $b_{i}$ are all distinct. In case $b_{i}=b_{i+1}$, one can attach $A_{\imath}$ to $A_{\imath+1}$ to obtain a new arc $A_{i} \cup A_{i+1}$ and then insert $O \otimes\left(A_{i} \cup A_{\imath+1}\right)$ into the widened gap of $\overline{O C_{1}\left(b_{i}\right)}$,

Since the cutting and inserting process preserves the length of the curve and the area of the cone, $\bar{C}$ satisfies 
( 5 )

$$
\begin{aligned}
& \text { Length }(\bar{C})=\text { Length }\left(C_{1}\right)+\sum_{\imath=2}^{k} \operatorname{Length}\left(A_{\imath}\right), \\
& \operatorname{Area}(O \otimes \bar{C})=\operatorname{Area}\left(O \otimes C_{1}\right)+\sum_{\imath=2}^{k} \operatorname{Area}\left(O \otimes A_{\imath}\right) .
\end{aligned}
$$

Here the curve $\bar{C}$ may have self intersections and the cone $O \otimes \bar{C}$ may have overlapping parts. In this case $\operatorname{Area}(O \otimes \bar{C})$ should count the multiplicity of the overlapping.

By (2), (3), (4), (5), we have

$$
\operatorname{Area}(p \times C)=\operatorname{Area}(O \otimes \bar{C}), \quad \text { Length }(C) \geqq \operatorname{Length}(\bar{C}) \text {. }
$$

Since $l(0)=l\left(\sum_{\imath=1}^{k} a_{\imath}\right)=0, \quad \bar{C}$ is a closed curve containing $O$. Therefore the conclusion follows from [5, Corollary 1$]$.

\section{Stationary varifolds}

Our purpose in this section is to prove the isoperimetric inequality for a stationary 2-dimensional varifold in $\boldsymbol{R}^{n}$. It is known that the restriction of a stationary varifold $V$ to the set of points of positive density is rectifiable $[3,1]$. And Allard proved that if the density of a stationary varifold is essentially bounded away from zero, then an open dense subset of the support of $V$ is a continuously differentiable submanifold of $\boldsymbol{R}^{n}$ [1]. Following [1] and [9], we briefly introduce varifolds in $\boldsymbol{R}^{n}$, define stationary varifolds and their generalized boundary, and derive an area estimate of a stationary varifold from the first variation formula.

m-dimensional varifolds in $\boldsymbol{R}^{n}$ are simply Radon measures on $G_{m}\left(\boldsymbol{R}^{n}\right)=$ $\boldsymbol{R}^{n} \times G(n, m)$, where $G(n, m)$ is the space of $m$-dimensional subspaces of $\boldsymbol{R}^{n}$. Given such an $m$-varifold $V$ in $\boldsymbol{R}^{n}$, there corresponds a Radon measure $\mu_{V}$ on $\boldsymbol{R}^{n}$ defined by

$$
\mu_{V}(A)=V\left(\pi^{-1}(A)\right), \quad A \subset \boldsymbol{R}^{n},
$$

where $\pi$ is the projection $(x, S) \mapsto x$ of $G_{m}\left(\boldsymbol{R}^{n}\right)$ onto $\boldsymbol{R}^{n}$. The mass $\boldsymbol{M}(V)$ of $V$ is defined by

$$
\boldsymbol{M}(V)=\mu_{V}\left(\boldsymbol{R}^{n}\right)=V\left(G_{m}\left(\boldsymbol{R}^{n}\right)\right) .
$$

If $M=\operatorname{spt} \mu_{V}$ is rectifiable, then $\mu_{V}=\mathscr{H}^{m}\left\llcorner\theta\right.$, where $\mathscr{H}^{m}$ is the $m$-dimensional Hausdorff measure, $\theta$ vanishes on $\boldsymbol{R}^{n} \sim M$ and is a positive locally $\mathscr{H}^{m}$-integrable function on $M . \quad \theta$ is called the multiplicity function of $\mu_{V}$. The support $M$ and multiplicity $\theta$ of $\mu_{V}$ completely determine $V$ when $V$ is rectifiable. So the varifold $V$ is also denoted by $v(M, \theta)$. When $\theta$ is integer valued almost everywhere, $V$ is called an integral varifold.

Let $f: \boldsymbol{R}^{n} \rightarrow \boldsymbol{R}^{n}$ be differentiable. Then we define the image varifold $f_{\#} V$ of $V$ by 


$$
f_{\#} V(A)=\int_{F^{-1}(A)} J_{S} f(x) d V(x, S), \quad A \subset G_{m}\left(\boldsymbol{R}^{n}\right),
$$

where $F: G_{m}\left(\boldsymbol{R}^{n}\right) \rightarrow G_{m}\left(\boldsymbol{R}^{n}\right)$ is defined by $F(x, S)=\left(f(x), d f_{x}(S)\right)$ and where

$$
J_{S} f(x)=\sqrt{\operatorname{det}\left(\left(d f_{x} \mid S\right)^{*_{0}}\left(d f_{x} \mid S\right)\right)}, \quad(x, S) \in G_{m}\left(\boldsymbol{R}^{n}\right),
$$

$\left(d f_{x} \mid S\right) *$ being the adjoint of $d f_{x} \mid S$.

The first varzation $\delta V$ of $V$ is a linear functional on the set $\mathscr{X}\left(\boldsymbol{R}^{n}\right)$ of continuous vector fields with compact support on $\boldsymbol{R}^{n}$, defined by

$$
\delta V(Y)=\left.\frac{d}{d t} \boldsymbol{M}\left(\phi_{t \#} V\right)\right|_{t=0},
$$

where $\left\{\phi_{t}\right\}_{-1<t<1}$ is any 1-parameter family of diffeomorphisms in $\boldsymbol{R}^{n}$ with $Y$ as the initial velocity vector field. Differentiation under the integral gives

$$
\delta V(Y)=\int_{G_{m}\left(\boldsymbol{R}^{n}\right)} \operatorname{div}_{S} Y(x) d V(x, S),
$$

where

$$
\operatorname{div}_{S} Y=\sum_{\imath=1}^{m}\left\langle\tau_{\imath}, D_{\tau_{i}} Y\right\rangle,
$$

$\tau_{1}, \cdots, \tau_{m}$ being an orthonormal basis for $S$, and $D$ the Euclidean connection.

$V$ is said to be stationary in $U$ if $\delta V(Y)=0$ for any $Y \in \mathfrak{X}\left(\boldsymbol{R}^{n}\right)$ with spt $|Y| \subset U$.

Now we want to define $\|\delta V\|$, the total variation measure of $\delta V$. Assume that $V$ has locally bounded first variation in $\boldsymbol{R}^{n}$, that is, for each $W \Subset \boldsymbol{R}^{n}$ there is a constant $c<\infty$ such that $|\delta V(Y)| \leqq c \sup |Y|$ for any $Y \in \mathscr{X}\left(\boldsymbol{R}^{n}\right)$ with spt $|Y| \subset W$. Then the Riesz representation theorem says that there exist a Radon measure $\|\delta V\|$ on $\boldsymbol{R}^{n}$ and a $\|\delta V\|$-measurable vector field $\nu$ on $\boldsymbol{R}^{n}$ such that $|\nu|=1\|\delta V\|$-a.e. and

$$
\delta V(Y)=\int_{R^{n}} \nu \cdot Y d\|\delta V\|,
$$

where $\|\delta V\|$ is characterized by

$$
\|\delta V\|(W)=\sup \left\{\delta V(Y): Y \in \mathscr{X}\left(\boldsymbol{R}^{n}\right),|Y| \leqq 1 \text {, and } \operatorname{spt}|Y| \subset W\right\}
$$

for any open $W \Subset \boldsymbol{R}^{n}$. Differentiating $\|\delta V\|$ with respect to $\mu_{V}$, we see that

$$
\frac{d\|\delta V\|}{d \mu_{V}}(x)=\lim _{\rho \rightarrow 0} \frac{\|\delta V\|\left(B_{\rho}(x)\right)}{\mu_{V}\left(B_{\rho}(x)\right)}
$$

exists $\mu_{V}$-a.e. and that

$$
\int_{R^{n}} \nu \cdot Y d\|\delta V\|=-\int_{R^{n}} \vec{H} \cdot Y d \mu_{V}+\int_{R^{n}} \nu \cdot Y d \sigma,
$$


where

$$
\begin{aligned}
& \vec{H}(x)=-\frac{d\|\delta V\|}{d \mu_{V}}(x) \nu(x), \quad \sigma=\|\delta V\|\llcorner Z, \\
& Z=\left\{x \in \boldsymbol{R}^{n}: \frac{d\|\delta V\|}{d \mu_{V}}(x)=\infty\right\}, \text { and } \mu_{V}(Z)=0 .
\end{aligned}
$$

Thus for $Y \in \mathscr{X}\left(\boldsymbol{R}^{n}\right)$ we can write

$$
\delta V(Y)=-\int_{R^{n}} \vec{H} \cdot Y d \mu_{V}+\int_{Z} \nu \cdot Y d \sigma .
$$

By analogy with the classical first variation formula for a smooth submanifold of $\boldsymbol{R}^{n}$, we call $\vec{H}$ the generalized mean curvature of $V, Z$ the generalized boundary of $V, \sigma$ the generalized boundary measure of $V$, and $\nu \mid Z$ the generalized unit conormal of $V$. We can easily see that $V$ is stationary in $U$ if and only if $\vec{H} \mid U=0$ and $Z \cap U=\emptyset$.

DEFINITION 2. (a) Let $V$ be an $m$-dimensional varifold of locally bounded first variation in $\boldsymbol{R}^{n}$ and $Z$ the generalized boundary of $V$ with the generalized boundary measure $\sigma$. Assume $Z$ is $(m-1)$-rectifiable. Let

$$
\psi(x)=\lim _{\rho \rightarrow 0} \frac{\sigma\left(B_{\rho}(x)\right)}{\mathscr{H}^{m-1}\left(Z \cap B_{\rho}(x)\right)}, \quad x \in Z .
$$

Then define the varifold boundary $\partial V$ of $V$ to be the varifold $v(Z, \phi)$. In other words, $\partial V$ is the $(m-1)$-dimensional rectifiable varifold with support $Z$ and multiplicity $\phi$. Clearly $\mu_{\partial V}=\sigma$.

(b) For an $m$-varifold $V=v(M, \theta)$, the varifold cone $p \times V$ from $p$ over $V$ is the $(m+1)$-varifold $v(p \otimes M, \bar{\theta})$, where $\bar{\theta}(y)=\theta(x)$ whenever $y$ lies on the line segment from $p$ to $x \in M$.

Example. Given a cube $I^{3}$ of volume 1 in $\boldsymbol{R}^{3}$, let $F$ be the union of the faces of $I^{3}, E$ the union of the edges of $I^{3}$. Define $V$ to be the 2-dimensional varifold with support $F$ and multiplicity 1 everywhere, i.e., $V=v(F, 1)$. Then one can see that i) the generalized mean curvature $\vec{H}$ of $V$ vanishes on $F \sim E$, ii) $E$ is the generalized boundary of $V$, iii) $\sigma=\left(\mathscr{H}^{1}\llcorner E)\llcorner\sqrt{2}\right.$ is the generalized boundary measure of $V$, and iv) the generalized unit conormal $\nu$ of $V$ makes an angle of 45 degrees with the outward unit normals to $F$ along $E$. It follows that $V$ is stationary in $\boldsymbol{R}^{3} \sim E$, the multiplicity of $\partial V$ is $\sqrt{2}$, i.e., $\partial V=v(E, \sqrt{2})$, and $p \times \partial V=v(p \times E, \sqrt{2}), p \in \boldsymbol{R}^{3}$. Moreover $\boldsymbol{M}(V)=6, \boldsymbol{M}(\partial V)=12 \sqrt{2}$, and if $p_{0}, p_{1}$ are the center of gravity and a vertex of $I^{3}$ respectively, $\boldsymbol{M}\left(p_{0} \otimes \partial V\right)=6$, $\boldsymbol{M}\left(p_{1} \otimes \partial V\right)=3+3 \sqrt{2}$.

In [5, Proposition 1] we have proved a volume estimate for minimal submanifolds in $\boldsymbol{R}^{n}$. We extend this estimate to stationary varifolds in $\boldsymbol{R}^{n}$ as 
follows.

THEOREM 1. Let $V$ be an m-varifold of locally bounded first variation in $\boldsymbol{R}^{n}$. If the generalized boundary $Z$ of $V$ is rectifiable and $V$ is stationary in $\boldsymbol{R}^{n} \sim Z$, then for any $p \in \boldsymbol{R}^{n}$

$$
\boldsymbol{M}(V) \leqq \boldsymbol{M}(p \rtimes \partial V)
$$

Proof. We see from (6) that

$$
\delta V(Y)=\int_{Z} \nu \cdot Y d \sigma, \quad Y \in \mathscr{X}\left(\boldsymbol{R}^{n}\right) .
$$

Take $Y$ to be the radial vector field defined by $Y(x)=x-p$. Then $Y$ is the initial velocity vector field of the 1-parameter family of homothetic expansions $\left\{\phi_{t}\right\}$ given by $\phi_{t}(x)=(1+t)(x-p)+p$. Hence

and so

$$
\boldsymbol{M}\left(\phi_{t \#} V\right)=(1+t)^{m} \boldsymbol{M}(V),
$$

$$
\delta V(Y)=\left.\frac{d}{d t}(1+t)^{m} \boldsymbol{M}(V)\right|_{t=0}=m \boldsymbol{M}(V)
$$

On the other hand, since $Z$ is rectifiable, $Z$ has tangent spaces almost everywhere and $\nu$ is normal to $Z$. Let $\eta(x)$ be a unit vector which is perpendicular to $Z$ at $x \in Z$ and lies in the subspace of $\boldsymbol{R}^{n}$ spanned by $Y(x)=x-p$ and the tangent space to $Z$ at $x$. Taking the negative of $\eta$ if necessary, one can assume $\eta \cdot Y \geqq 0$. It is not difficult to see that

$$
\nu \cdot Y \leqq \eta \cdot Y \text {. }
$$

Let $r(x)=|Y(x)|$. Then $d r$ is the 1-form dual to the unit radial vector field $Y /|Y|$. Hence

$$
\left.m \boldsymbol{M}(V)=\int_{Z} \nu \cdot Y d \boldsymbol{\sigma} \leqq \int_{Z} \eta \cdot Y d \boldsymbol{\sigma}=\int_{Z} Y\right\lrcorner(d r \wedge d \sigma)=m \boldsymbol{M}(p \rtimes \partial V) .
$$

Remark. In Theorem 1 we can see that $\boldsymbol{M}(V)=\boldsymbol{M}(p \times \partial V)$ if and only if $\nu=\eta$ everywhere on $Z$, or equivalently, $\nu(x)$ lies in the subspace spanned by $Y(x)$ and the tangent space to $Z$ at every $x \in Z$. This is the case in the example preceding Theorem 1 when $p=$ the center of gravity of $I^{3}$. More generally, for a stationary integral varifold $V$ arising from the boundary (=faces) of a regular polyhedron $U, \boldsymbol{M}(V)=\boldsymbol{M}(p \otimes \partial V)$, provided $p$ is the center of gravity of $U$.

LEMMA 3. Let $W=v(Z, \phi)$ be a rectifiable 1-varifold in $\boldsymbol{R}^{n}$ with $\phi \geqq 1$ and let $p$ be a point in $Z$. If $Z$ is a compound Jordan curve, then

$$
4 \pi \boldsymbol{M}(p \times W) \leqq \boldsymbol{M}(W)^{2} .
$$


Proof. Lemma 2 is a special case of this lemma with $Z=C$ and $\phi=1$. So this lemma will be proved by Lemma 2 if we can construct a compound Jordan curve $C$ in $\boldsymbol{R}^{n}$ with $p \in C$ such that

$$
\operatorname{Area}(p \otimes C)=\boldsymbol{M}(p \otimes W), \quad \text { Length }(C) \leqq \boldsymbol{M}(W) .
$$

Let $\gamma_{1}, \cdots, \gamma_{k} \subset Z$ be the curves as defined in the proof of Lemma 2, and let $J$ be the union of end points of $\gamma_{2}, \cdots, \gamma_{k}$. Then $Z \sim J$ is a union of curves none of which is a loop. Still let $\gamma_{1}, \cdots, \gamma_{m}$ denote the components of $Z \sim J$. And let $p_{\imath}^{1}, p_{i}^{2}$ be the end points of $\gamma_{\imath}$. Define $W_{\imath}$ to be the part of $W$ corresponding to $\gamma_{\imath}$, i.e., $W_{\imath}=v\left(\gamma_{\imath}, \psi \mid \gamma_{\imath}\right)$. Parametrize $\gamma_{\imath}$ by $\theta, 0 \leqq \theta \leqq a_{\imath}$. Let $l(\theta)=$ $\operatorname{dist}\left(p, \gamma_{i}(\theta)\right)$ and $\phi(\theta)=\left(\phi \mid \gamma_{2}\right)(\theta)$. Assume, as in the proof of Lemma 2, that $\theta$ is the angle parameter so that $l(\theta)$ satisfies

$$
\boldsymbol{M}\left(p \times W_{\imath}\right)=\frac{1}{2} \int_{0}^{a_{\imath}} l(\theta)^{2} \phi(\theta) d \theta, \quad \boldsymbol{M}\left(W_{\imath}\right)=\int_{0}^{a_{\imath}} \sqrt{l^{2}+\left(\frac{\partial l}{\partial \theta}\right)^{2}} \phi(\theta) d \theta .
$$

Define a new parameter $\phi$ of $\gamma_{\imath}, 0 \leqq \phi \leqq b_{i}$, such that $\phi=f(\theta)=\int_{0}^{\theta} \phi(t) d t, f\left(a_{\imath}\right)$ $=b_{i} . \quad f$ is monotone increasing and its inverse function exists. Then $l(\theta)=$ $l\left(f^{-1}(\phi)\right)$ and hence

$$
\begin{aligned}
& \boldsymbol{M}\left(p \otimes W_{\imath}\right)=\frac{1}{2} \int_{0}^{b_{i}} l\left(f^{-1}(\phi)\right)^{2} d \phi, \\
& \boldsymbol{M}\left(W_{\imath}\right)=\int_{0}^{b_{i}} \sqrt{l^{2}+\left(\frac{\partial l}{\partial \phi}\right)^{2}\left(\frac{\partial \phi}{\partial \theta}\right)^{2}} d \phi \geqq \int_{0}^{b_{i}} \sqrt{l^{2}+\left(\frac{\partial l}{\partial \phi}\right)^{2}} d \phi,
\end{aligned}
$$

where we used the fact that $\partial \phi / \partial \theta=\phi \geqq 1$. In view of the similarity of (8) to the formulas for the area and arc length in polar coordinates, one can easily construct a curve $\bar{\gamma}_{i}$ from $p_{i}^{1}$ to $p_{i}^{2}$ parametrized by the angle parameter $\phi$ such that $l\left(f^{-1}(\phi)\right)=\operatorname{dist}\left(p, \bar{\gamma}_{i}(\phi)\right)$ and

$$
\operatorname{Area}\left(p \times \bar{\gamma}_{\imath}\right)=\boldsymbol{M}\left(p \times W_{\imath}\right), \quad \text { Length }\left(\bar{\gamma}_{\imath}\right) \leqq \boldsymbol{M}\left(W_{\imath}\right) \text {. }
$$

One can further impose on $\bar{\gamma}_{2}$ the condition that $\gamma_{i} \cap J \subset \bar{\gamma}_{i} \cap J$. Define $C=$ $\cup_{i=1}^{m} \bar{\gamma}_{\imath}$. Clearly $C$ is a compound Jordan curve, and $p \in C$ since $l=0$ at $p$. Summing up (9) for $\imath$, we get (7) as desired.

THEOREM 2. Suppose that $V$ is a 2-varifold of locally bounded first variation in $\boldsymbol{R}^{n}$, the generalized boundary $Z$ of $V$ is rectifiable, and $V$ is stationary in $\boldsymbol{R}^{n} \sim Z$. If the multiplicity of $\partial V$ is $\geqq 1$ and $Z$ is a compound Jordan curve, then

$$
4 \pi \boldsymbol{M}(V) \leqq \boldsymbol{M}(\partial V)^{2} .
$$

Proof. Apply Theorem 1 and Lemma 3.

We conjecture that the theorem above can be extended in three ways: i) The theorem should hold without the hypothesis on the multiplicity of $\partial V$ if 
the multiplicity of $V$ is assumed to be 1 a.e.; ii) The optimal case (equality) should occur only when spt $V$ is a disk; iii) The theorem should be true even when the generalized boundary $Z$ of $V$ is a disjoint union of compound Jordan curves. The difficulty lies in obtaining the angle estimate: The angle of $Z$ viewed from a point of spt $V \sim Z$ should be not smaller than $2 \pi$. If the singular set of spt $V$ is the union of differentiable curves (like J. Taylor's soap-film-like surfaces [11]) and if spt $V$ is regular up to $Z$, then the angle estimate easily follows (see [5, Remarks]). Once we have the angle estimate, we can apply the arguments of [5, Theorem 1] and prove the isoperimetric inequality for $V$ if $Z$ is radially connected from a point of spt $V \sim Z$.

In case the multiplicity of $\partial V$ is less than 1 , one can modify the theorem as follows.

COROLLARY 1. Let $V$ be a 2-varifold of locally bounded first variation in $\boldsymbol{R}^{n}$ such that $V$ is stationary outside the rectifiable generalized boundary $Z$. Write $\partial V=v(Z, \theta)$ and define $\bar{\partial} V=v(Z, \bar{\theta}), \bar{\theta}=\max \{\theta, 1\}$. If $Z$ is a compound Jordan curve, then

$$
4 \pi \boldsymbol{M}(V) \leqq \boldsymbol{M}(\bar{\partial} V)^{2} .
$$

\section{Area minimizing flat chains $\bmod k$}

In this section we derive a different type of sharp isoperimetric inequality for certain area minimizing flat chains $\bmod k$. Roughly speaking, flat chains, or currents, are obtained by assigning an orientation to the tangent space of varifolds. First let us briefly define currents and related terminology.

Let $\mathscr{D}^{m}$ be the space of smooth differential $m$-forms with compact support in $\boldsymbol{R}^{n}$. An $m$-dimensional current in $\boldsymbol{R}^{n}$ is a continuous linear functional on $\mathscr{D}^{m}$. The set of such $m$-currents will be denoted $\mathscr{D}_{m}$. Any oriented $m$-dimensional rectifiable set $M$ may be viewed as a current $T_{M}$ in the following way. Let $\vec{S}(x)$ denote the unit $m$-vector associated with the oriented tangent space to $M$ at $x$. Then for any differential $m$-form $\omega$, define

$$
T_{M}(\omega)=\int_{M}\langle\vec{S}(x), \omega\rangle d \mathscr{H}^{m} .
$$

Furthermore, we will allow $T_{M}$ to carry a positive integer multiplicity $\theta(x)$, and define

$$
T_{M, \theta}(\boldsymbol{\omega})=\int_{M}\langle\vec{S}(x), \omega\rangle \theta(x) d \mathscr{H}^{m} .
$$

Motivated by the classical Stokes theorem, we are led to define the boundary $\partial T \in \mathscr{D}_{m-1}$ of an $m$-current $T$ by

$$
\partial T(\omega)=T(d \omega), \quad \omega \in \mathscr{D}^{m-1} .
$$

Again motivated by the example above, $T_{M}$, we define the mass of $T, \boldsymbol{M}(T)$, 
for $T \in \mathscr{D}_{m}$ by

$$
\boldsymbol{M}(T)=\sup \left\{T(\omega):|\omega| \leqq 1, \omega \in \mathscr{D}^{m}\right\},
$$

where $|\omega|=\sup _{x \in R^{n}}\langle\boldsymbol{\omega}(x), \omega(x)\rangle^{1 / 2}$. The support of a current $T$, spt $T$, is the complement in $\boldsymbol{R}^{n}$ of the largest open set on which $T=0 . T$ is called a rectifiable current if spt $T$ is rectifiable. The mass of a rectifiable current is just the Hausdorff measure of the associated rectifiable support (counting multiplicities). The integer multiplicity rectifiable currents $T_{M, \theta}$ as defined in (10) are characterized by the property that they agree, to within a set of arbitrarily small $\mathscr{H}^{m}$ measure, with $m$-dimensional $C^{1}$ singular chains with integer coefficients. Notice that one can associate $T_{M, \theta}$ with the integer multiplicity varifold $V=v(M, \boldsymbol{\theta})$ in $\boldsymbol{R}^{n}$.

$\Omega_{m}$ denotes the set of integer multiplicity rectifiable $m$-currents in $\boldsymbol{R}^{n}$. And $g_{m}^{k}$ denotes the space of $m$-dimensional rectifiable flat chains modulo $k$ whose boundaries are also rectifiable flat chains modulo $k$, that is,

$$
\mathfrak{g}_{m}^{k}=\left\{T: T \in \mathscr{R}_{m} / k \mathscr{R}_{m}, \partial T \in \mathscr{R}_{m-1} / k \mathscr{R}_{m-1}\right\}
$$

(see $[7,4.2 .26])$. We write the same notations spt, $\partial, \boldsymbol{M}$ for flat chains $\bmod k$ as we do for currents. One says $T \in \mathcal{G}_{m}^{k}$ is area minımizıng if

$$
\boldsymbol{M}(T) \leqq \boldsymbol{M}(S) \text { for every } S \in \mathcal{G}_{m}^{k} \text { with } \partial S=\partial T .
$$

Defininion 3. Let $Y^{k} \subset \boldsymbol{R}^{3}$ be the union of $k$ great semicircles on a sphere meeting at the north and south poles at equal angles of $2 \pi / k$. Define $y_{2}^{k} \subset g_{2}^{k}$ to be the set of 2-dimensional flat chains $T \bmod k$ in $\boldsymbol{R}^{n}$ with multiplicity 1 almost everywhere such that spt $\partial T$ is homeomorphic to $Y^{k}$ and the associated varifold $V=v(\operatorname{spt} T, \theta)$ is locally of bounded first variation in $\boldsymbol{R}^{n}$.

THEOREM 3. Suppose that $T$ is a 2-dimensional area minımızıng flat chain $\bmod k$ in $y_{2}^{k}$. If $C_{1}, C_{2}, \cdots, C_{k}$ are the curves that constitute spt $\partial T$ and have common end points $p, p^{\prime}$, then

$$
2 \pi \boldsymbol{M}(T) \leqq \sum_{\imath=1}^{k} \text { Length }\left(C_{\imath}\right)^{2} .
$$

And equality holds of and only if spt $T$ is the unon of $k$ flat half disks meetıng each other along the common diameter.

LEMMA 4. Let $T \in \mathrm{y}_{2}^{k}$ be a 2-dimensional area minımizing flat chain $\bmod k$ and $V$ the varifold associated with $T$. Then spt $\partial V \subset$ spt $\partial T$ and the multıplicity $\phi$ of $\partial V$ is less than or equal to 1 almost everywhere on $\operatorname{spt} \partial T$.

Proof of Lemma 4. Since $T$ is area minimizing, $V$ is stationary in $\boldsymbol{R}^{3} \sim$ spt $\partial T$. Hence spt $\partial V \subset \operatorname{spt} \partial T$. Now let us write $\partial V=v(\operatorname{spt} \partial T, \phi)$ and suppose $\phi>1$ on a set $U \subset$ spt $\partial T$ with $\mathscr{H}^{1}(U)>0$. As stated in the previous section, the generalized unit conormal $\nu$ of $V$ is $\|\delta V\|$-measurable. Hence we can approxi- 
mate $-\nu \mid U$ by a smooth vector field $Y$ with compact support with respect to $L^{1}$ norm on $U$. Extend $Y$ to a smooth vector field $\bar{Y}$ on $\boldsymbol{R}^{n}$. Then by (6) $\delta V(\bar{Y})$ can be made sufficiently close to $-\sigma(U)$. Note that

$$
\sigma(U)=\int_{U} \phi d \mathscr{H}^{1}>\mathscr{H}^{1}(U) .
$$

Let $\left\{\phi_{t}\right\}_{-1<t<1}$ be a 1-parameter family of diffeomorphisms in $\boldsymbol{R}^{n}$ with $\bar{Y}$ as the initial velocity vector field and with $\left\{x \in \operatorname{spt} \partial T: \phi_{t}(x) \neq x\right\} \subset U$. Then

$$
\boldsymbol{M}\left(\phi_{t \#} V\right)=\boldsymbol{M}(V)-t[\sigma(U)+\varepsilon]+O\left(t^{2}\right)
$$

for some sufficiently small $|\varepsilon|$. Now define a 2-dimensional rectifiable set $D_{t}$, $t>0$, to be the union of the line segments from $\phi_{0}(x)$ to $\phi_{t}(x)$ for all $x \in U$. Clearly

$$
\operatorname{Area}\left(D_{t}\right)=t\left[\mathscr{K}^{1}(U)+\bar{\varepsilon}\right]+O\left(t^{2}\right),
$$

with $|\bar{\varepsilon}|$ sufficiently small. By giving a suitable orientation to $D_{t}$, one can make $D_{t}$ into a current, still denoted $D_{t}$, such that $S_{t}=\phi_{t \#} T+D_{t}$ is a flat chain $\bmod k$ with $\partial S_{t}=\partial T$. Moreover we have from (12), (13)

$$
\boldsymbol{M}\left(S_{t}\right)=\boldsymbol{M}\left(\phi_{t \#} V\right)+\boldsymbol{M}\left(D_{t}\right)=\boldsymbol{M}(T)+t\left[-\boldsymbol{\sigma}(U)+\mathscr{T}^{1}(U)-\varepsilon+\bar{\varepsilon}\right]+O\left(t^{2}\right) .
$$

Therefore we can deduce from (11) that for some $t, \boldsymbol{M}\left(S_{t}\right)<\boldsymbol{M}(T)$, which is a contradiction.

Proof of Theorem 3. By Theorem 1 and Lemma 4 we have

$$
\boldsymbol{M}(T) \leqq \boldsymbol{M}(p \times \partial V) \leqq \sum_{\imath=1}^{k} \operatorname{Area}\left(p \times C_{\imath}\right) .
$$

Since each $p \times C_{\imath}$ is flat, one can develop it on a plane and apply Lemma 1(a) to get

Hence

$$
2 \pi \operatorname{Area}\left(p \times C_{\imath}\right) \leqq \text { Length }\left(C_{\imath}\right)^{2} .
$$

$$
2 \pi \boldsymbol{M}(T) \leqq \sum_{\imath=1}^{k} \text { Length }\left(C_{\imath}\right)^{2} .
$$

Here equality holds if and only if $\boldsymbol{M}(T)=\sum_{\imath=1}^{k} \operatorname{Area}\left(p \otimes C_{\imath}\right)$ and if each $p C_{\imath}$ can be developed onto a half disk. So if equality holds, then the flat chain $\bmod k, T_{M}$, associated with $M=\bigcup_{\imath=1}^{k} p \times C_{\imath}$ is area minimizing. Let $r(x)=|x-p|$. Then the slice of $T_{M}$ by $r$ at $\rho,\left\langle T_{M}, r, \rho\right\rangle$, is a 1-dimensional flat chain $\bmod k$ in $\partial B_{\rho}(p)$ for almost all $\rho>0$. Furthermore $\left\langle T_{M}, r, \rho\right\rangle$ is locally length minimizing, i.e., $\left\langle T_{M}, r, \rho\right\rangle\llcorner D$ is length minimizing for any sufficiently small domain $D$ in $\partial B_{\rho}(p)$. Hence $\operatorname{spt}\left\langle T_{M}, r, \rho\right\rangle$ is the union of part of great circles in $\partial B_{\rho}(p)$. Therefore $M$ is a polyhedral set and each $C_{\imath}$ is a planar curve. Now let $\bar{C}_{\imath}$ be the semicircle centered at $p$, ending at $p^{\prime}$, and of radius $\left|p^{\prime}-p\right|$ such that the half disk $\bar{D}_{\imath}$ determined by $\bar{C}_{\imath}$ (i.e., $\partial \bar{D}_{\imath} \supset \bar{C}_{\imath}$ ) contains the half 
disk $D_{\imath}$ determined by $C_{\imath}$. Define a new flat chain $\bmod k S$ such that $\operatorname{spt} S=$ spt $T \cup\left(\cup_{i=1}^{k}\left(\bar{D}_{i} \sim D_{\imath}\right)\right)$ and spt $\partial S=\bigcup_{i=1}^{k} \bar{C}_{\imath}$. Then we have $\boldsymbol{M}(S)=4 \boldsymbol{M}(T)$ since $\boldsymbol{M}(T)=\sum_{\imath=1}^{k} \operatorname{Area}\left(D_{\imath}\right)$. Note that $\bar{C}_{\imath}$ is similar to $C_{\imath}$ and that Length $\left(\bar{C}_{\imath}\right)=$ 2 Length $\left(C_{\imath}\right)$. Hence one can easily see that $S$ also is area minimizing. Let $p^{\prime \prime}$ be the other end point of $\bar{C}_{\imath}$. Along the open line segment of $\overline{p p^{\prime \prime}}$, the density of $S$ is $k / 2$. Then by the upper semicontinuity of the density, the density of $S$ at $p$ is at least $k / 2$. And the monotonicity of the mass ratio (a flat chain version of $[7,5.4 .3]$ ) says that

$$
\Theta^{2}(S, p, r)=\boldsymbol{M}\left(S\llcorner B(p, r)) / \pi r^{2}\right.
$$

is a monotonically nondecreasing function of $r$. But for $d=\operatorname{dist}\left(p, p^{\prime}\right)$, $\Theta^{2}(S, p, d)=k / 2$, and $\lim _{r \rightarrow 0} \Theta^{2}(S, p, r) \geqq k / 2$. Hence $\Theta^{2}(S, p, r)$ is a constant function and spt $S$ is a cone. Therefore spt $T=\bigcup_{\imath=1}^{k} p \otimes C_{\imath}$ whenever equality holds in the isoperimetric inequality.

Let $Y$ be a union of three half disks meeting each other along their common diameter at equal angles of 120 degrees. Let $T$ be the intersection with the unit ball $B_{1}(O)$ of an infinite cone from $O$ through the 1-skeleton of a regular tetrahedron with its center of mass at $O$. In [11] J. Taylor proved that the disk, $Y$, and $T$ are the only three cones that are area minimizing under Lipschitz maps leaving the boundary fixed. In view of this fact we raise the following problem as an analogue of Theorem 3 .

Open Problem. Suppose that $V$ is a 2 -varifold with multiplicity 1 almost everywhere and is locally of bounded first variation in $\boldsymbol{R}^{n}$ such that $V$ is stationary outside the rectifiable boundary $\operatorname{spt} \partial V$. Suppose also that $\operatorname{spt} V$ is homeomorphic to $T$. Let $C_{1}, C_{2}, \cdots, C_{6} \subset$ spt $\partial V$ be the curves that constitute spt $\partial V$ and lie between 4 junctions of spt $\partial V$. Show that

$$
\left[2 \cos ^{-1}\left(-\frac{1}{3}\right)\right] \boldsymbol{M}(V) \leqq \sum_{\imath=1}^{6} \text { Length }\left(C_{\imath}\right)^{2}
$$

where equality holds if and only if spt $V$ is a homothetic expansion (or contraction) of $T$.

\section{REFERENCES}

[1] W. K. Allard, First variation of a varifold, Ann. of Math. (2), 95 (1972), 417-491.

[2] F.J. Almgren, Jr., Optimal isoperimetric inequalities, Indiana Univ. Math. J., 35 (1986), 451-547.

[3] F. J. Almgren, JR., The theory of varifolds, Mimeographed, Princeton University, 1965.

[4] T. CARLEMAN, Zur Theorie der Minimalflächen, Math. Z., 9 (1921), 154-160.

[5] J. CHOE, The isoperimetric inequality for a minimal surface with radially connected boundary, Ann. Scuola Norm. Sup. Pisa C1. Sc1. (4), 17 (1990), 583-593. 
[6] J. Choe and R. Gulliver, The sharp isoperimetric inequality for minimal surfaces with radially connected boundary in hyperbolic space, Invent. Math., 109 (1992), 495-503.

[7] H. FEDERER, Geometric Measure Theory, Springer, Berlin, 1969.

[8] C.C. Hsiung, Isoperimetric inequalities for two-dimensional Riemannian manifolds with boundary, Ann. of Math. (2), 73 (1961), 213-220.

[9] L. Simon, Lectures on Geometric Measure Theory, Australian National University, 1983.

[10] M. SpIVAK, A Comprehensive Introduction to Differential Geometry, vol. 4, Publish or Perish, Berkeley, 1979.

[11] J.E. TAYLOR, The structure of singularities in soap-bubble-like and soap-filmlike minimal surfaces, Ann. of Math. (2), 103 (1976), 489-539.

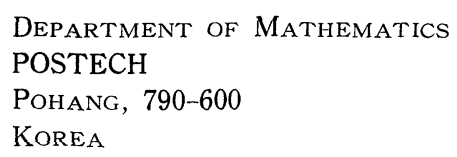

